\title{
Distinguishing Arbitrary Multipartite Basis Unambiguously Using Local Operations and Classical Communication
}

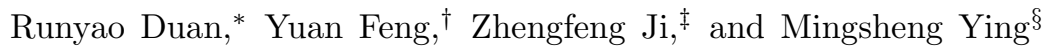 \\ State Key Laboratory of Intelligent Technology and Systems, \\ Department of Computer Science and Technology, Tsinghua University, Beijing, China, 100084
}

(Dated: November 4, 2018)

\begin{abstract}
We show that an arbitrary basis of a multipartite quantum state space consisting of $K$ distant parties such that the $k$ th party has local dimension $d_{k}$ always contains at least $N=\sum_{k=1}^{K}\left(d_{k}-1\right)+1$ members that are unambiguously distinguishable using local operations and classical communication (LOCC). We further show this lower bound is optimal by analytically constructing a special product basis having only $N$ members unambiguously distinguishable by LOCC. Interestingly, such a special product basis not only gives a stronger form of the weird phenomenon "nonlocality without entanglement", but also implies the existence of locally distinguishable entangled basis.
\end{abstract}

PACS numbers: 03.67.-a, 03.67.Mn, 03.65.Ud, 03.67.Hk

Suppose we are given a quantum system whose state is secretely chosen from a finite set of pre-specified unit vectors. Because of the limitations of quantum mechanics, the state of the system can be identified with certainty if and only if these vectors are mutually orthogonal. Remarkably, when these vectors are nonorthogonal but linearly independent, the state of the system can also be identified unambiguously with some nonzero success probability [1]. Consequently, any orthogonal (or nonorthogonal) basis always can be exactly (respectively, unambiguously) discriminated when there is no restrictions on the quantum measurements one can perform.

However, the situation becomes very complicated when the given quantum system is shared by a finite number of distant parties, where each party holds a piece of the whole system and can perform local operations and classical communication (LOCC) only [2]. The number of states one can locally discriminate significantly decreases. Perhaps the most surprising discovery in this field is due to Bennett and collaborators in Ref. [3], where they exhibited nine $3 \otimes 3$ orthogonal product states that are not exactly distinguishable using LOCC, thus initiated the study of the famous phenomenon "nonlocality without entanglement". Another fundamental contribution was made by Walgate et al. in Ref. [4], where it was demonstrated that any two multipartite orthogonal pure states, whether entangled or not, can always be exactly discriminated using LOCC. Since then, many interesting works on the local distinguishability of quantum states have been done and two kinds of results have been reported [5, 6, 7, 8, 9, 10, 11, 12, 13, 14, 15, 16]. The first kind of results follows Ref. [3] to show that certain set of quantum states cannot be discriminated by LOCC. The second kind of results is, similar to Ref. [4], to identify sets of states that are locally distinguishable. In particular, an orthonormal basis that is unambiguously distinguishable by LOCC must be a product basis, as shown by Horodecki et al. [13]. Another very interesting result is recently obtained by Bandyopadhyay and
Walgate in Ref. [16], where they showed that among any three linearly independent pure states, there always exists one state that can be unambiguously determined using LOCC.

In this Letter we consider the local distinguishability of a general nonorthogonal multipartite basis. Our main result is that arbitrary multipartite basis has at least $N$ members that are unambiguously distinguishable by LOCC. This lower bound is shown to be tight by explicitly constructing a product basis which has only $N$ members that are unambiguously distinguishable by LOCC (Theorem 2). The significance of our result is that it gives a universal tight lower bound on the number of the locally unambiguously distinguishable members in arbitrary multipartite basis and thus provides new insight into the local distinguishability of a multipartite basis. We further obtain an equivalence between locally distinguishable entangled basis (DEB) (basis having entangled states as members) and indistinguishable product basis (IPB) (basis having product states as members) (Theorem 3), and present explicit constructions of such special basis. In particular, the existence of locally indistinguishable product basis gives a stronger form of "nonlocality without entanglement". Furthermore, these results yield the following counterintuitive conclusion about the relations among orthogonality, entanglement, and local distinguishability: Orthogonal states are not always more easily locally distinguishable than nonorthogonal ones, sometimes less orthogonality and more entanglement (or vice versa) may enhance the local distinguishability of a set of quantum states. Most notably, in proving our main results, we introduce a useful notion named unextendible bases and employ it as a tool to study the local distinguishability of quantum states. As a byproduct, we obtain an interesting connection between the local unambiguous distinguishability and unextendible bases (Theorem 1).

Let us now begin to introduce the notion of unextendible bases, which is a generalization of the orthogonal 
unextendible product bases that was first introduced by Bennett et al. [5] and was then extensively studied in Ref. [6]. The key difference is that in Refs. [5, [6] only orthogonal product states are considered while here arbitrary states (entangled or unentangled) may be involved. Let $\mathcal{H}=\otimes_{k=1}^{K} \mathcal{H}_{k}$ be a multipartite quantum system with $K$ parties. Each $\mathcal{H}_{k}$ is a $d_{k}$-dimensional Hilbert space. Sometimes we use the notation $d_{1} \otimes \cdots \otimes d_{K}$ for $\mathcal{H}$. Let $S$ be a subset of $\mathcal{H}$. Then $\operatorname{span}(S)$ is the subspace spanned by the vectors in $S$, and $S^{\perp}$ represents the orthogonal complement of $S$.

Definition 1 (unextendible bases): Let $S=$ $\left\{\Psi_{1}, \cdots, \Psi_{m}\right\}$ be a collection of $m$ linearly independent quantum states on $\mathcal{H}$. $S$ is said to be an unextendible bases (UB) if $S^{\perp}$ contains no product state; otherwise $S$ is said to be extendible. Furthermore, $S$ is said to be a genuinely unextendible bases(GUB) if it is unextendible and any proper subset of $S$ is extendible. In particular, when $S$ is a collection of product states, we use the notions unextendible product bases (UPB) and genuinely unextendible product bases (GUPB) instead of unextendible bases and genuinely unextendible bases, respectively.

It follows directly from the above definition that any orthogonal UPB is necessarily a GUPB. However, when nonorthogonal states are considered, there does exist UPB that is not a GUPB. See Example 2 below for an explicit instance.

A notion closely related to unextendbile bases is the completely entangled subspace introduced by Parthasarthy [17]: $S$ is a UB implies $S^{\perp}$ is completely entangled; conversely, $S$ is completely entangled indicates that any basis of $S^{\perp}$ constitutes a UB. Such a correspondence suggests that some works that have been done for completely entangled subspaces may be useful in studying UB.

The following lemma provides a lower bound on the size of a UB.

Lemma 1: Any UB on $\mathcal{H}$ must have at least $N=$ $\sum_{k=1}^{K}\left(d_{k}-1\right)+1$ members.

Proof: Let $S$ be a UB on $\mathcal{H}$. Then the cardinality of $S$ is just the dimension of $\operatorname{span}(S)$. For simplicity, we write directly $\operatorname{dim}(S)$ for the cardinality of $S$. Then $\operatorname{dim}(S)+\operatorname{dim}\left(S^{\perp}\right)=\operatorname{dim}(\mathcal{H})$. By the definition, $S^{\perp}$ is a completely entangled subspace of $\mathcal{H}$. We notice that it has been proven by Parthasarthy [17] that the maximal dimension of a completely entangled subspace of $\mathcal{H}$ is $\operatorname{dim}(\mathcal{H})-N$. Applying this result we have that $\operatorname{dim}(S)=$ $\operatorname{dim}(\mathcal{H})-\operatorname{dim}\left(S^{\perp}\right) \geq N$. That completes the proof.

It is well known that the construction of orthogonal UPB is an extremely difficult task [5, 6]. Interestingly, constructing nonorthogonal UB and UPB is very simple. We shall need the following counting lemma which is essentially due to Bennett et al. [5]:

Lemma 2: Let $S=\left\{\left|\psi_{j}\right\rangle=\otimes_{k=1}^{K}\left|\varphi_{k j}\right\rangle: 1 \leq j \leq N\right\}$ be a collection of product states on $\mathcal{H}$. If for each $1 \leq k \leq$
$K$, any subset of $\left\{\left|\varphi_{k j}\right\rangle: 1 \leq j \leq N\right\}$ with $d_{k}$ members spans $\mathcal{H}_{k}$, then $S$ is a GUB for $\mathcal{H}$.

Assisting with Lemma 2, we are now in a position to present an explicit construction of GUPB with minimal cardinality.

Example 1 (minimal UPB): For each $1 \leq k \leq K$, let $\left\{|j\rangle: 0 \leq j \leq d_{k}-1\right\}$ be an orthonormal basis for $\mathcal{H}_{k}$. For each $1 \leq k \leq K$ and $x \in \mathcal{C}$, define $\left|\varphi_{k}(x)\right\rangle=$ $N_{k}(x)^{-1} \sum_{j=0}^{d_{k}-1} x^{j}|j\rangle$, where $N_{k}(x)=\sqrt{\sum_{j=0}^{d_{k}-1}|x|^{2 j}}$ is the normalized factor. For simplicity, let $\left|\varphi_{k}(\infty)\right\rangle=$ $\lim _{x \rightarrow \infty}\left|\varphi_{k}(x)\right\rangle=\left|d_{k}-1\right\rangle$, and let $\mathcal{C}_{+}=\mathcal{C} \cup\{\infty\}$. A key property of $\left|\varphi_{k}(x)\right\rangle$ is that for any pairwise different elements $x_{0}, \cdots, x_{d_{k}-1}$ from $\mathcal{C}_{+},\left|\varphi_{k}\left(x_{0}\right)\right\rangle, \cdots,\left|\varphi_{k}\left(x_{d_{k}-1}\right)\right\rangle$ are linearly independent and thus form a basis for $\mathcal{H}_{k}$. This can be seen from the nonsingularity of Van der Monde matrix $\left[x_{m}^{n}\right], 0 \leq m, n \leq d_{k}-1$. (Note that the nonsingularity is also valid when one of $x_{m}$ is $\infty$ ).

For $\lambda=\left(\lambda_{1}, \cdots, \lambda_{K}\right) \in \mathcal{C}_{+}^{K}$, we define $|\psi(\lambda)\rangle=$ $\left|\varphi_{1}\left(\lambda_{1}\right)\right\rangle \otimes \cdots \otimes\left|\varphi_{K}\left(\lambda_{K}\right)\right\rangle$. Take an index set $I=$ $\left\{\lambda^{(1)}, \cdots, \lambda^{(N)}\right\} \subseteq \mathcal{C}_{+}^{K}$ such that any two elements in $I$ are entrywise distinct, i.e., $\lambda_{k}^{(m)} \neq \lambda_{k}^{(n)}$ for any $1 \leq m<$ $n \leq N$ and $1 \leq k \leq K$. Then the set $\{|\psi(\lambda)\rangle: \lambda \in I\}$ is a UPB on $\mathcal{H}$.

By Lemma 2, we only need to show that for each $1 \leq$ $k \leq K$, any $d_{k}$ members of $\left\{\left|\varphi_{k}\left(\lambda_{k}^{(j)}\right)\right\rangle: 1 \leq j \leq N\right\}$ are linearly independent and thus form a basis for $\mathcal{H}_{k}$. This clearly holds by the pairwise distinctness of $\left\{\lambda_{k}^{(j)}: 1 \leq\right.$ $j \leq N\}$ and by the special form of $\left|\varphi_{k}().\right\rangle$.

In particular, take $K=2, d_{1}=d_{2}=2, I=$ $\{(0,0),(1,1),(\infty, \infty)\}$, then $\{|0\rangle|0\rangle,|+\rangle|+\rangle,|1\rangle|1\rangle\}$ is a minimal UPB on $2 \otimes 2$.

A special case of the above construction ( $\lambda$ satisfies $\lambda_{1}=\cdots=\lambda_{K}$ ) was first given by Parthasarthy and then was considerably studied by Bhat [17]. Unfortunately, their method can only yield $N$ linearly independent vectors. The above construction is much more general and can be used to construct a product basis for $\mathcal{H}$, as we will see latter.

We shall present a connection between local unambiguous distinguishability and unextendible bases. The following lemma, which is a simplified version of a more general result due to Chefles [11], indicates that the condition for unambiguous discrimination is much more complicated when only LOCC operations are allowed. Recently an alternative proof was obtained in Ref. [16].

Lemma 3: Let $S=\left\{\Psi_{1}, \cdots, \Psi_{m}\right\}$ be a collection of $m$ quantum states on $\mathcal{H}$. Then $S$ can be unambiguously discriminated by LOCC if and only if for each $1 \leq k \leq$ $m$, there exists a product detecting state $\left|\psi_{k}\right\rangle$ such that $\left\langle\Psi_{j} \mid \psi_{k}\right\rangle=0$ for $j \neq k$ and $\left\langle\Psi_{k} \mid \psi_{k}\right\rangle \neq 0$.

It should be noted that the proof of Lemma 3 is not constructive. So far there is no feasible way to determine the existence of the product detecting states [11, 16]. Consequently, for a given set of quantum states, it is highly nontrivial to determine the local distinguishabil- 
ity of these states.

Now we are ready to investigate the local distinguishability of a UB. It has been shown by Bennett et al. 5] that the members of an orthogonal UPB cannot be exactly discriminated by LOCC. But clearly they are unambiguously distinguishable by LOCC, as we can always choose the state itself as the corresponding detecting state. Interestingly, this property holds for any GUB.

Theorem 1: A UB can be unambiguously discriminated by LOCC if and only if it is a GUB.

Proof: Let $S=\left\{\Psi_{1}, \cdots, \Psi_{m}\right\}$ be a GUB. Consider the set $S_{k}=S-\left\{\Psi_{k}\right\} . S_{k}$ is a proper subset of $S$ and thus is extendible. So there exists a product state $\left|\psi_{k}\right\rangle$ such that $\left\langle\psi_{k} \mid \Psi_{j}\right\rangle=0$ for any $j \neq k$. We claim that $\left\langle\psi_{k} \mid \Psi_{k}\right\rangle \neq 0$. Otherwise, it holds $\left|\psi_{k}\right\rangle \in S^{\perp}$. This contradicts the assumption that $S$ is unextendible. Hence $\left|\psi_{k}\right\rangle$ is exactly a product detecting state for $\Psi_{k}$. That proves the unambiguous distinguishability of $S$.

Now assume $S$ is a UB but not a GUB. So there is some proper subset $S^{\prime}$ of $S$ that is unextendible. In other words, $S^{\prime} \subset S$ is also a UB. Take $\Psi \in S-S^{\prime}$. It is easy to see there cannot be a product state $|\psi\rangle$ that is orthogonal to the vectors in $S^{\prime}$. Thus $\Psi$ cannot have a product state as its detector, and cannot be identified with a nonzero probability from $S-\{\Psi\}$.

Let us check some interesting consequences of Theorem 1. Suppose $S$ is a subspace spanned by a GUB with dimension $N$. Then any basis for $S$ is also a GUB. Thus it follows from Theorem 1 that any basis for $S$ is unambiguously distinguishable by LOCC. Interestingly, recently Watrous found another kind of special bipartite subspace having no basis exactly distinguishable by LOCC [14].

Suppose now that $S$ is a UB but not a GUB. By the above theorem, $S$ is not unambiguously distinguishable by LOCC. On the other hand, $S$ always contains a proper subset, say $S^{\prime}$, that is a GUB. Thus $S^{\prime}$ can be unambiguously discriminated by LOCC. An important special case is when $S$ is a basis for $\mathcal{H}$. Combining Theorem 1 with Lemma 1, we arrive at the following central result:

Theorem 2: Any basis for $\mathcal{H}$ has at least $N$ members unambiguously distinguishable by LOCC. Furthermore, the lower bound $N$ is tight in the sense there exists a basis for which any $N+1$ members cannot be unambiguously discriminated by LOCC.

Remark: In the case when $K=1$ (unipartite), $N$ is reduced to $d_{1}$ and we recover the well-known result that any basis can be unambiguously discriminated by unconstrained measurements [1].

Proof: Notice that any basis for $\mathcal{H}$ is a UB and thus contains a GUB. By Lemma 1 and Theorem 1, such a GUB has a size at least $N$ and is unambiguously distinguishable by LOCC. That completes the proof of the first part. Now we consider the second part. We shall give an explicit construction of the basis that has only $N$ members unambiguously distinguishable by LOCC. For $x \in \mathcal{C}$, define

$$
\Psi(x)=\left|\psi\left(x^{d_{2} \cdots d_{K}}, \cdots, x^{d_{K}}, x\right)\right\rangle,
$$

where $|\psi()$.$\rangle is the same as in Example 1. After some$ algebraic manipulations we have

$$
\Psi(x)=N(x)^{-1} \sum_{j=0}^{d-1} x^{j}|j\rangle_{\mathcal{H}},
$$

where $d=d_{1} \cdots d_{K}, j=\sum_{k=1}^{K} j_{k}\left(d_{k+1} \cdots d_{K}\right),|j\rangle_{\mathcal{H}}=$ $\otimes_{k=1}^{K}\left|j_{k}\right\rangle_{\mathcal{H}_{k}}, 0 \leq j_{k} \leq d_{k}-1$, and $N(x)$ is a normalized factor. On the one hand, take $d$ pairwise distinct complex numbers $x_{1}, \cdots, x_{d}$ such that

$$
x_{m}^{\prod_{j=k+1}^{K} d_{j}} \neq x_{n}^{\prod_{j=k+1}^{K} d_{j}}, 1 \leq m<n \leq d, 1 \leq k \leq K .
$$

Then by the nonsingularity of Von der Monde matrix, we have that $S=\left\{\Psi\left(x_{1}\right), \cdots, \Psi\left(x_{d}\right)\right\}$ is a basis for $\mathcal{H}$. On the other hand, by Example 1, any subset of $S$ with $N$ members constitutes a GUB for $\mathcal{H}$. So any subset $S^{\prime}$ of $S$ with $N+1$ members is a UB but not a GUB. The indistinguishability of $S^{\prime}$ follows from Theorem 1.

One may naturally expect a stronger form of Theorem 2: Any $N$ linearly independent states of $\mathcal{H}$ can be unambiguously discriminated by LOCC. Unfortunately, this cannot hold even for $2 \otimes 2$ states, as we have the following result: For any $\mathcal{H}=\otimes_{k=1}^{K} \mathcal{H}_{k}$, there always exist three orthogonal pure states $\Psi_{1}, \Psi_{2}$, and $\Psi_{3}$ that are not unambiguously distinguishable by LOCC. A simple instance is as follows (see Ref. [16] for a similar construction):

$$
\Psi_{1}=|x\rangle, \Psi_{2,3}=\frac{|0\rangle^{\otimes K} \pm|1\rangle^{\otimes K}}{\sqrt{2}},
$$

where $x$ is a $K$-bit string such that $x \neq 0^{K}, 1^{K}$. We can easily verify that $\Psi_{2(3)}$ cannot have a product detecting state. Thus $\Psi_{1}, \Psi_{2}$, and $\Psi_{3}$ are not unambiguously distinguishable by LOCC. This is an example of $K$ qubits. Obviously it can also be treated as an example on any composite quantum system consisting of $K$ parties.

Notice that any $d+1$ orthogonal maximally entangled states on $d \otimes d$ cannot be exactly distinguishable by LOCC [9]. Interestingly, by Theorem 2 we conclude immediately at least $2 d-1$ maximally entangled states can be unambiguously discriminated by LOCC. An explicit construction is as follows. Let $\left\{\Phi_{m, n}: 0 \leq m, n \leq d-1\right\}$ be the canonical maximally entangled basis on $d \otimes d$, where

$$
\Phi_{m, n}=1 / \sqrt{d} \sum_{k=0}^{d-1} \omega^{k n}|k\rangle|k+m \bmod d\rangle, \omega=e^{\frac{2 \pi i}{d}} .
$$

Let $S=\left\{\Phi_{m, n}: m n=0\right\}$. We claim that $S$ is unambiguously distinguishable by LOCC. This is due to 
the fact that $\operatorname{span}(S)$ can also be spanned by a minimal GUPB, i.e., any $2 d-1$ states of the following set $\{|m\rangle|m\rangle: 0 \leq m \leq d-1\} \cup\{|\bar{m}\rangle|\bar{m}\rangle: 0 \leq m \leq d-1\}$, where $|\bar{m}\rangle=1 / \sqrt{d} \sum_{k=0}^{d-1} \omega^{k m}|k\rangle$. Here we have employed Example 1 and Theorem 1.

Let us now consider the following question: What kind of basis for $\mathcal{H}$ can be unambiguously discriminated by LOCC? Furthermore, can we find a locally DEB? Surprisingly, we shall show there do exist a DEB. Indeed we have a more general result: An equivalence between a DEB and an IPB. Then the existence of DEB follows directly from the existence of IPB. To state this equivalence, we need the notion of reciprocal basis. Suppose $S=\left\{\Psi_{1}, \cdots, \Psi_{d}\right\}$ is a basis for $\mathcal{H}$. For each $1 \leq k \leq d$, we can uniquely determine the reciprocal state $\widetilde{\Psi}_{k}$ of $\Psi_{k}$ as follows: $\left\langle\widetilde{\Psi}_{k} \mid \Psi_{j}\right\rangle=0$ for any $j \neq k$. Then the reciprocal basis for $S$, denoted by $\widetilde{S}$, is just the collection of $\widetilde{\Psi}_{k}$.

Theorem 3: Let $S$ be a basis for $\mathcal{H}$. Then $S$ is unambiguously distinguishable by LOCC if and only if $\widetilde{S}$ is a product basis. Furthermore, $S$ is a DEB if and only if $\widetilde{S}$ is an IPB.

Proof: This result is essentially due to Lemma 3 and the following fact: The reciprocal basis of $\widetilde{S}$ is just $S$ (up to some unimportant phase factors), i.e., $\widetilde{\widetilde{S}}=S$.

We have presented an analytical construction of IPB in the proof of Theorem 2. By the above theorem, a DEB can be obtained by taking its reciprocal basis. An illustrative example of DEB on $2 \otimes 2$ is as follows:

Example 2: Consider the following four states: $\Psi_{1}=$ $|0\rangle|0\rangle, \Psi_{2}=|1\rangle|1\rangle, \Psi_{3}=|+\rangle|+\rangle, \Psi_{4}=\left|i_{+}\right\rangle\left|i_{-}\right\rangle$, where $\left|i_{ \pm}\right\rangle=1 / \sqrt{2}(|0\rangle \pm i|1\rangle) . S=\left\{\Psi_{1}, \Psi_{2}, \Psi_{3}, \Psi_{4}\right\}$ is a product basis for $2 \otimes 2$ and any proper subset of $S$ with three members is a GUPB. Thus $S$ is an IPB. By Theorem 3, the reciprocal basis $\widetilde{S}$ is a DEB. The members of $\widetilde{S}$ are calculated as follows:

$$
\begin{aligned}
& \widetilde{\Psi}_{1}=1 / \sqrt{2}|00\rangle-1 / 2 \Phi_{+}+1 / 2 i \Phi_{-}, \\
& \widetilde{\Psi}_{2}=1 / \sqrt{2}|11\rangle-1 / 2 \Phi_{+}+1 / 2 i \Phi_{-},
\end{aligned}
$$

and $\widetilde{\Psi}_{3,4}=\Phi_{ \pm}=1 / \sqrt{2}|01\rangle \pm 1 / \sqrt{2}|10\rangle$. These states are unambiguously distinguishable by LOCC as for each $1 \leq k \leq 4, \Psi_{k}$ is just a product detecting state for $\widetilde{\Psi}_{k}$.

In conclusion, we introduce the notion of unextendible bases and employ it to study the local distinguishability of multipartite quantum states. In particular, a tight lower bound on the number of locally unambiguously distinguishable members of an arbitrary basis is presented. We also obtain an equivalence between DEB and IPB, and exhibit analytical constructions of such special basis. This equivalence motivates us to consider the relation among orthogonality, entanglement, and local distinguishability. Roughly speaking, neither orthogonality nor entanglement can uniquely determine the distinguishablity of a basis. There may exist a tradeoff: More orthogonality and less entanglement (or vice versa) would sometimes enhance the local distinguishability of quantum states. A challenging problem left is to obtain similar bounds in the context of exact LOCC discrimination. (Partial results have been obtained in Ref. [15]).

We thank J.-X. Chen, G.-M. Wang, Z.-H. Wei, and C. Zhang for helpful discussions. We also thank Dr. S. Bandyopadhyay for pointing out a typographical error in the previous version of this Letter. This work was partly supported by the Natural Science Foundation of China (Grant Nos. 60621062 and 60503001), the Hi-Tech Research and Development Program of China (863 project) (Grant No. 2006AA01Z102), and the National Basic Research Program of China (Grant No. 2007CB807901).

* Electronic address: dry@tsinghua.edu.cn

$\dagger$ Electronic address: feng-y@tsinghua.edu.cn

¥ Electronic address: jizhengfeng98@mails.tsinghua.edu.cn

$\S$ Electronic address: yingmsh@tsinghua.edu.cn

[1] A. Chefles, Phys. Lett. A 239, 339 (1998); L.-M. Duan and G.-C. Guo, Phys. Rev. Lett. 80, 4999 (1998).

[2] A. Peres and W. K. Wootters, Phys. Rev. Lett. 66, 1119 (1991).

[3] C. H. Bennett, D. P. DiVincenzo, C. C. Fuchs, T. Mor, E. Rains, P. W. Shor, J. A. Smolin, and W. K. Wootters, Phys. Rev. A 59, 1070 (1999).

[4] J. Walgate, A.J. Short, L. Hardy, and V. Vedral, Phys. Rev. Lett. 85, 4972 (2000).

[5] C. H. Bennett, D. P. Divincenzo, T. Mor, P. W. Shor, Phys. Rev. Lett. 82, 5385 (1999).

[6] D. P. DiVincenzo, T. Mor, P. W. Shor, J. A. Smolin, and B.M. Terhal, Comm. Math. Phys. 238, 379 (2003); N. Alon and L. Lovasz, J. of Combinatorial Theory, Ser. A. 95, 169 (2001); J. Niset and N. J. Cerf, Phys. Rev. A 74, 052103 (2006).

[7] S. Virmani, M. F. Sacchi, M. B. Plenio, and D. Markham, Phys. Lett. A 288, 62 (2001); Y.-X. Chen and D. Yang, Phys. Rev. A 64, 064303 (2001); Phys. Rev. A 65, 022320 (2002); M. Hillery and J. Mimih, Phys. Rev. A 67, 042304 (2003); Z. Ji, H. Cao, and M. Ying, Phys. Rev. A 71, 032323 (2005); A. Acín, E. Bagan, M. Baig, Ll. Masanes, and R. Muñoz-Tapia, Phys. Rev. A 71, 032338 (2005); Y. Ogata, J. Phys. A: Math. Gen. 39, 3059 (2006).

[8] B. M. Terhal, D. P. DiVincenzo, and D. W. Leung, Phys. Rev. Lett. 86, 5807 (2001); D. P. DiVincenzo, D. W. Leung, and B. M. Terhal, IEEE Trans. Inf. Theory 48, 580 (2002); T. Eggeling and R. F. Werner, Phys. Rev. Lett. 89, 097905 (2002).

[9] S. Ghosh, G. Kar, A. Roy, A. Sen(De), and U. Sen, Phys. Rev. Lett. 87, 277902 (2001); Phys. Rev. A 65, 062307 (2002); H. Fan, Phys. Rev. Lett. 92, 177905 (2004); M. Nathanson, J. Math. Phys. 46, 062103 (2005).

[10] J. Walgate and L. Hardy, Phys. Rev. Lett. 89, 147901 (2002); M.-Y. Ye, W. Jiang, P.-X. Chen, Y.-S. Zhang, Z.-W. Zhou, G.-C. Guo, quant-ph/0608040

[11] A. Chefles, Phys. Rev. A 69, 050307(R) (2004).

[12] P.-X. Chen and C.-Z. Li, Phys. Rev. A 70, 022306 (2004); 
S. De Rinaldis, Phys. Rev. A 70, 022309 (2004).

[13] M. Horodecki, A. Sen(De), U. Sen, and K. Horodecki, Phys. Rev. Lett. 90, 047902 (2003).

[14] J. Watrous, Phys. Rev. Lett. 95, 080505 (2005).

[15] M. Hayashi, D. Markham, M. Murao, M. Owari, and S. Virmani, Phys. Rev. Lett. 96, 040501 (2006).
[16] S. Bandyopadhyay and J. Walgate, quant-ph/0612013

[17] K. R. Parthasarathy, Proc. Indian Acad. Sci. 114, 365 (2004), quant-ph/0405077, B. V. R. Bhat, quant-ph/0409032. 